\title{
Artículo
}

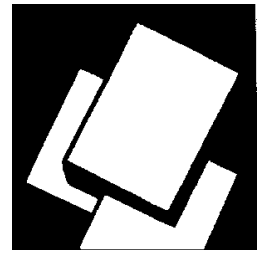

\section{Estudio comparativo de la satisfacción laboral en el personal de administración}

\section{A comparative study of work satisfaction among public servants}

PILAR ALONSO MARTÍN1

\section{RESUMEN}

Partiendo del Modelo de las carencias del trabajador (Herzberg, 1959) se plantea analizar si existen diferencias en la percepción de la satisfacción laboral. Es un estudio descritivo-transversal con una muestra de 80 sujetos pertenecientes al Personal de Administración y Servicios de un Universidad pública. Se ha utilizado el cuestionario de Satisfacción en el trabajo (Warr, Cokk y Wall, 1979). Los resultados muestran un nivel más alto de satisfacción laboral en las mujeres, los trabajadores de más edad y los con un mayor nivel de antigüedad y con un contrato de interino. Se concluye que son los factores externos, más concretamente, las relaciones sociales con los mandos superiores los que producen menor satisfacción en esta muestra, independientemente de las distintas variables medidas. Se hace necesario acciones concretas para mejorar la comunicación vertical.

\section{ABSTRACT}

In line with Herzberg's model (Herzberg, 1959) this paper raises the question of the existence of differences in the perception of work satisfaction. It is a descriptive cross sectional study made with a sample of 80 public servants belonging to the administration and services in a public university. The Work Satisfaction Questionnaire (Warr, cokk and Wall, 1979) was used as the measurement tool. Results show that females, older people, more

${ }^{1}$ Departamento de Psicología y de la Educación. Facultad de Ciencias de la Educación. Campus «El Carmen», Universidad de Huelva. pilar.alonso@dpsi.uhu.es 
senior employees and temporary workers had a higher level of work satisfaction. It is concluded that it is external factors, such as leader-subordinate exchange what lowers satisfaction regardless of other variables. Specific actions should be taken in order to improve vertical communication.

\section{PALABRAS CLAVE}

Satisfacción laboral, Sexo, Antigüedad, Tipo de contrato, Factores externos y factores internos.

\section{KEY WORDS}

Job satisfaction, Gender, Seniority, Contract type, External and internal factors. 
El tema de la satisfacción laboral es de gran interés porque nos indica la habilidad de la organización para satisfacer las necesidades de los trabajadores y porque muchas evidencias demuestran que los trabajadores insatisfechos faltan a trabajar con más frecuencia y suelen renunciar más, mientras que los empleados satisfechos gozan de mejor salud y viven más años.

Desde el punto de vista de la psicología, el trabajo proporciona salud física y mental, entretenimiento, prestigio, contactos sociales y es un fuente de realización personal (Gamero, 2003). Por ello, muchas veces la insatisfacción laboral tiene efectos nocivos sobre la productividad del individuo. Existen datos disponibles que muestran la importancia de los aspectos laborales en la salud física y mental de los trabajadores (Faragher, Cass y Cooper, 2005). Se ha observado que la insatisfacción laboral se asocia de forma importante a trastornos mentales comunes (Quintero, Biela, Barrera y Campo, 2007). Los trabajadores insatisfechos, manifiestan con mayor frecuencia síntomas ansiosos y depresivos (Newbury, Kamali, 2001; Wieclaw, Agerbo, Mortensen y Bonde, 2005). Asimismo, se ausentan un gran número de días de trabajo (Virtanen, Kimimaki, Elovainio, Batear y Ferrie, 2003) y asisten, por lo general, más veces a consulta médica (Harmon, Scotti, Behson, Farias, Petzel y Neuman, 2003).

En la actualidad no existe una definición unánimemente aceptada sobre el concepto de satisfacción laboral. Es más, en muchas ocasiones cada autor elabora una nueva definición para el desarrollo de su propia investigación (Harpaz, 1983). En primer lugar, existen una serie de definiciones que hacen referencia a la satisfacción laboral como estado emocional sentimientos o respuestas afectivas (Crites, 1969; Locke, 1976; Smith, Kendall y Hullin, 1969). Un segundo grupo de autores considera que la satisfacción laboral va más allá de las respuestas afectivas o emocionales y de ahí la importancia que ésta tiene en las conductas laborales. Estos autores conciben la satisfacción laboral como una actitud generalizada ante el trabajo (Arnold, Robertson y Cooper, 1991; Beer, 1964; Bravo, 1992; Griffin y Bateman, 1986; Harpaz, 1983; Peiró, 1984; Salancik y Pfeffer, 1977). Otros autores han considerado diferentes facetas en la satisfacción laboral (Bravo, 1992; Cook, Hepwoorth, Wall y Warr, 1981; Peiró, 1984; Vroom, 1964). No obstante, muchas de esas facetas únicamente difieren entre sí en cuanto a su denominación o etiqueta pues el contenido al que hacen referencia es muy similar. Así, por ejemplo, la dimensión de satisfacción con la retribución (Smith, Kendall y Hulin, 1969; Warr y Routledge, 1969) ha sido etiquetada por otros autores como satisfacción con las recompensas extrínsecas (Schmitt y Loher, 1984) o como satisfacción extrínseca (Weiss, Davis, England y Lofquist, 1967) pero definiéndose todas ellas del mismo modo.

En general, las facetas de satisfacción más mencionadas tienen que ver con la satisfacción con el contenido del trabajo (interés intrínseco del trabajo, la variedad, las oportunidades de aprendizaje o la dificultad), con el salario, con las condiciones de trabajo (horario, descansos, condiciones ambientales) y con la empresa.

Ante esta gran variedad de facetas, Herzberg (1959) señala que existen facto- 
res de motivación intrínsecos del trabajo, vinculados directamente con la satisfacción laboral, tales como el trabajo mismo, los logros, el reconocimiento recibido por el desempeño, la responsabilidad y los ascensos. Los factores extrínsecos del trabajo, que no pueden ser controlados o modificados directamente por el trabajador, están relacionados con la higiene, las políticas de organización, la supervisión, la disponibilidad de recursos, el salario y la seguridad.

Aunque el estudio sistemático de la naturaleza y las causas de la satisfacción laboral no comienza hasta los años 30 , ya antes algunos autores estudiaron diversas actitudes de los empleados ante distintos aspectos del trabajo. El problema de la reducción de la fatiga fue uno de los primeros en tratarse. Al hilo de éste, se investigaron cuestiones como las referentes a las horas de trabajo y los descansos (Vernon, 1921; Wyatt, 1927, citados en Peiró, 1984). En el caso de España, las investigaciones sobre la satisfacción laboral eran escasas hasta hace poco tiempo. Son ya clásicas la encuesta de Castillo (1968) aplicada a los trabajadores andaluces y la de Tezanos, Lopez Aparicio, Rodríguez, y Domínguez (1973) aplicado a los empleados de la banca .

Los estudios empíricos han identificado muchos factores que condicionan el nivel de satisfacción en sus puestos de trabajo. Además de las características personales y de los rasgos de las empresas y de los puestos de trabajo que son incorporadas en la mayoría de las investigaciones, algunos autores han puesto el acento en el salario (Clark y Oswald, 1996 y Sloane y Williams, 2000), las posibilidades de promoción laboral (Leonardi y Sloane, 2000), las condiciones físicas del trabajo (Renaud, 2002) o la seguridad laboral (Wooden y Warren, 2004).

A tenor de lo anteriormente expuesto nuestro estudio se centra en determinar, en una muestra del Personal de Administración y Servicio de la Universidad de Huelva, si existen diferencias en la satisfacción laboral en función del sexo, escala administrativas, tipo de contrato y antigüedad en la universidad, teniendo como referencia el Modelo de las carencias del trabajador y la definición de insatisfacción (Herzberg, 1959).

\section{MÉTODO}

El diseño empleado ha sido transversal y descriptivo

\section{Sujetos}

El muestreo fue intencionado y por conglomerados, pues necesitábamos una muestra representativa de las distintas variables a estudiar.

La población la constituyen 220 sujetos entre funcionarios (45\%), laborales $(35 \%)$ e interinos $(20 \%)$

Nuestra muestra está formada por 80 sujetos pertenecientes al personal de administración y servicios de la universidad de Huelva, de los cuales el 32,5\% corresponde a hombre y el $67,5 \%$ a mujeres; un $49 \%$ son funcionario, un $31 \%$ laboral y un $20 \%$ interino. La media es de 40,2 años (DT=5,48) estando el $52 \%$ en la franja de edad de menos de 35 años, un $38 \%$ entre 36 y 45 y un $10 \%$ tiene más de 45 años. 
En la variable estado civil, el porcentaje más alto corresponde a casados/as, con un $64 \%$, seguido de solteros/as con un $32 \%$ y sólo un $4 \%$ corresponden a separados/as o divorciados/as. La mitad de nuestra muestra tiene hijos, desde 1 a 3 siendo la moda 2 hijos.

Un 55\% llevan trabajando más de 8 en la Universidad y un $45 \%$ menos de 8 años.

\section{Instrumentos:}

Se ha empleado la escala de "Satisfacción en el trabajo" de Warr, Cokk y Wall (1979), la cual establece una dicotomía de factores y está diseñada para observar tanto los aspectos intrínsecos como los extrínsecos de las condiciones de trabajo (Pérez y Fidalgo, NTP 394, 1994). A partir de un estudio piloto y de dos investigaciones en trabajadores de la industria manufacturera en Inglaterra, se definió la escala con 16 ítems.

Está formada por dos subescalas:

- Subescala de factores intrínsecos: aborda aspectos como el reconocimiento laboral, responsabilidad, promoción,aspectos relativos al contenido de la tarea, etc. Está formada por los ítems 2, 4, 6, 8, 10, 12 y 14 .

- Subescala de factores extrínsecos: aborda aspectos relativos a la organización del trabajo como el horario, el salario, las condiciones físicas del trabajo. Esta escala lo constituyen 8 ítems que son el 1, 3, 5, 7, 9, 11, $13 \mathrm{y}$ 15 .

El ítem 16 mide la satisfacción general en el trabajo.
Es una escala aditiva, en la cual la puntuación total se obtiene sumando las puntuaciones dados por cada sujeto en cada uno de los 16 ítems, asignando un valor de 1 a $M u y$ insatisfecho y correlativamente hasta asignar un valor de 7 a Muy satisfecho. La puntuación total de la escala oscila entre 15 y 105 , de manera que a mayor puntuación refleja mayor satisfacción general.

Además también se aplicó un pequeño cuestionario con ítems sociedomográficos: sexo, edad, estado civil, escala laboral, tipo de contrato y antigüedad en la Universidad.

\section{Procedimiento}

Inicialmente se contactó con el Personal de Administración y Servicios (P.A.S.) que reunían las características del muestro y se les expuso los objetivos de la investigación y el compromiso de entregar los resultados de la investigación si así lo pidieran. Igualmente se les pidió que nos informasen de qué día y en qué momento les vendría mejor la aplicación de dicho cuestionario.

La recogida de información se llevó a cabo a través del cuestionario, que fue cumplimentada de forma anónima por el P.A.S. de la Universidad de Huelva.

La sala donde se procedió a la administración de los cuestionarios fue en el mismo puesto de trabajo, procurando en todo momento interrumpir lo menos posible su jornada laboral.

\section{ANÁLISIS Y DISCUSIÓN \\ DE RESULTADOS}

\section{Comparación de medias por sexo}

Cuando se comparan las medias de los 
ítems de la escala de satisfacción laboral en función al sexo se observa lo siguiente:

- Las mujeres manifiestan un mayor grado de satisfacción en todos los ítems exceptuando la oportunidad para promocionar (factor interno) $y$ el horario de trabajo (factor externo). Esto puede ser debido a la dificultad que tienen las mujeres para conciliar la vida laboral y la familiar y puede satisfactorios en las mujeres que en los hombres.

En el único ítem que existen diferencias significativas es en el salario donde las mujeres siguen puntuando más alto que los hombres (tabla 1).

Según Gamero Burón (2003), el salario medio masculino es un $30 \%$ superior al femenino, fundamentalmente por la

Tabla 1. Comparación de medias del salario

\begin{tabular}{|l|l|l|}
\hline SEXO & $\mathrm{X}$ & D.T \\
\hline Varón & 3,73 & 1,017 \\
\hline Mujer & 4,56 & 1,031 \\
\hline & $\mathrm{t}(78)=2,259 \mathrm{p}=.027$ & \\
\hline
\end{tabular}

ser más satisfactorio un tipo de horario más flexible para poder adecuarse a ambas facetas; al igual que al tener que realizar más tareas que los hombres, es más difícil poder promocionar, pues si es por concurso-oposición o por oposición, tienen menos tiempo para dedicarlo a la preparación de la oposición

Nuestros datos coinciden con los estudios de Quin, Stances y McColloug (1974) quienes señalan que las mujeres valoran más que los hombres los factores de "comodidad" en el trabajo: número de horas, distribución de la jornada, lugar de trabajo, residencia, etc, todos ellos orientados a buscar la conciliación entre trabajo y familia; al igual que el trabajo realizado por Lucas Marín (1981) donde señala que, aunque los diversos estudios sobre la influencia del sexo en la satisfacción laboral no han aportado datos excesivamente esclarecedores de la relación entre ambas, los resultados obtenidos han sido más mayor incidencia entre la mujeres de la jornada a tiempo parcial (16\% frente a $8 \%$ en los hombres). Algunos hechos indican que tal diferencia podría no ser el resultado de un discriminación salarial directa contra las mujeres, sino de la segregación ocupacional, tanto vertical como horizontal, y sin olvidar el "techo de cristal", tal y como señalan Cuadrado y Morales (2007) en su investigación sobre dicho obstáculo. Así el porcentaje de mujeres en puesto de responsabilidad es aproximadamente la mitad que el de los hombres (19\% frente a $10 \%)$. Además las mujeres declaran con una frecuencia algo mayor que los hombres que su salario se corresponde con el asignado por el mercado para el tipo de puesto que ocupan y que están sobreeducadas.

- Tanto en la suma de los factores internos, externos y total, las mujeres obtienen unas medias más altas, aunque las diferencias no son significativas (tabla 2). 
Tabla 2. Comparación de factores internos/externos y total por sexo

\begin{tabular}{|l|c|c|c|}
\hline & F. interno & F. externos & Total \\
\hline Varón & 29,2 & 35,1 & 64,6 \\
\hline Mujer & 31,5 & 37,6 & 69,1 \\
\hline
\end{tabular}

Numerosas investigaciones apoyan la existencia de discriminación negativa contra la mujer en el mercado laboral, que se traduce en segregación ocupacional, menor nivel salarial y de oportunidades de promoción, comportamiento de acoso en el trabajo, mayores tasas de despido, etc. También cuando se analizan indicadores generales de bienestar subjetivo, las mujeres muestran una peor situación que los hombres (Clark y Oswald, 1994). Sin embargo, la mayoría de las encuestas usadas en los análisis de satisfacción laboral revelan que las mujeres están más satisfechas con su trabajo que los hombres.

¿Cómo puede explicarse este paradójico resultado?. En la literatura se han investigado cuatro hipótesis, basadas en la presencia de diferenciales por género en las característcas personales o de los trabajos, en los "gustos" o valores laborales, en las tasas de participación o en la expectativas laborales (Clark, 1997; Sloane y Williams, 2000). Igualmente Long (2005) considera que estas diferencias pueden deberse a las distintas expectativas que desarrollan hombres y mujeres en relación con su carrera profesional. Algunos autores concluyen, con fundamento en evidencia indierecta, que el diferencial en satisfacción se debe a que las mujeres trabajadoras, en general, esperan menos de sus trabajos que los hombres. Otros investigadores han puesto mayor énfasis en que esas discrepancias en satisfacción son el resultado de los distintos esquemas valorativos respecto a las características de los empleos (Groot y Maasen van den Brink, 1998; Sloane y Williams, 2000); estas investigaciones señalan que las mujeres valoran más las relaciones personales, mientras que los hombres lo hacen con la carrera profesional y el dinero. Por último, la exploración de esta cuestión en un marco internacional, apoya la idea de que la paradoja de la satisfacción por género parece ser un fenómeno, principalmente anglosajón (Souza-Posa y Souza-Posa, 2000; Kaiser, 2002).

\section{Comparación de medias por edad}

Teniendo en cuenta esta variable se observa:

- El grupo de edad que está más satisfecho es el de mayor de 45 años.

- Analizando por edades:

- el grupo de menos de 35 años puntúa más bajo que los otros grupo de edad en condiciones fisicas, libertad para escoger el método de trabajo, reconocimiento de una buena labor, salario, oportunidad para emplear sus capacidades, relaciones sociales entre dirección y empleados, oportunidad para pomocionar, forma en que se dirige la sección, atención que se le presta a sus sugerencias, el horario de trabajo, las diferentes actividades que realiza en su trabajo y la estabilidad laboral. 
Estudio comparativo de la satisfacción laboral en el personal de administración

- El grupo de 36-45 años sólo puntúa bajo en el ítem externo de los compañeros de trabajo.

- El grupo de más de 45 años puntúa más bajo y por tanto tiene menos satisfacción respecto al jefe inmediato y la cantidad de responsabilidad que tiene en su trabajo.

- Pero sólo los ítems condiciones físicas en el trabajo y oportunidad de promocionar obtienen diferencias significativas entre los tres grupos de edad.
Pero son contrarios a lo encontrado por Gamero Burón (2003) que encontró que la posibilidad de ser promocionado en la empresa sólo encuentra valoración positiva entre los jóvenes; la oportunidad de progresar en la empresa resulta más relevante al incio de la trayectoria profesional que cuando se ha alcanzado un nivel determinado. Esto puede ser debido a la nueva remuneración que va asociada a la nueva situación, a la vez que le supone una reafirmación personal para el joven.

- Las medias de los factores internos, externos y total mantienen la misma

Tabla 3. Comparación de medias por edad

\begin{tabular}{|l|l|l|}
\hline \multicolumn{1}{|c|}{ EDAD } & Condiciones físicas & Oport. promocionar \\
\hline Menor de 35 años & 4,07 & 3,22 \\
\hline 36-45 años & 4,55 & 4,06 \\
\hline Mayor de 45 & 5,5 & 4,25 \\
\hline & $\mathrm{F}(2,77)=4,04 \mathrm{p}=.021$ & $\mathrm{~F}(2,77)=3,41 \mathrm{p}=.038$ \\
\hline
\end{tabular}

Esto coincide con los datos de Robina Ramírez (2002) donde concluye que a medida que aumenta la edad más motivados están para implicarse en los traba- tónica anterior, siendo el grupo de más edad el que obtiene puntuaciones más altas, pero dichas diferencias no son significativas (tabla 4 ).

Tabla 4. Comparación de factores internos/externos y total por edades

\begin{tabular}{|l|c|c|c|}
\hline \multicolumn{1}{|c|}{ EDAD } & F. interno & F. externos & Total \\
\hline Menor de 35 años & 29.3 & 35.6 & 65 \\
\hline 36-45 & 32.4 & 37.5 & 70 \\
\hline Mayor de 45 & 31.3 & 39.8 & 71.2 \\
\hline
\end{tabular}

jos que desarrollan, valorando en mayor medida los factores internos de la relación laboral. Al igual que los datos encontrados por Lucas Marín (1981) donde la relación entre edad y satisfacción es positiva a partir de intervalos de 31-40 años, alcanzando el máximo de satisfacción en el intervalo previo a la jubilación.
Algunas investigaciones han abordado la importancia del significado del trabajo en función de los distintos intervalos de edad, así Morse y Weiss (1955, citado en Robina, op. cit) analizan algunas variables como la necesidad de trabajar en las hipotéticas circunstancias de disponer de unas cantidades económicas que hagan innecesario acudir al trabajo; según los resulta- 
dos obtenidos, el $90 \%$ de los trabajadores en el intervalo de edad de 21-34 años seguirían trabajando, a partir de aquí empezaría a descender hasta un $61 \%$ lo que indica una relación inversa entre la motivación en el trabajo y la edad.

\section{Comparación de medias por tipo de contrato}

Respecto al tipo de contrato tenemos lo siguiente:

- Los funcionarios: obtienen menos satisfacción en el trato con los compañero y en la relación con el jefe más inmediato.
- Sí existen diferencias significativas teniendo en cuenta el tipo de contrato en los siguientes ítems: reconocimiento de una buena labor $\left(\mathrm{F}_{(2,77)}=3,764\right.$, $\mathrm{p}=.028)$, salario $\left(\mathrm{F}_{(2,77)}=14,875\right.$, $\mathrm{p}=.000)$, oportunidad para emplear sus capacidades $\left(\mathrm{F}_{(2,77)}=9,593, \mathrm{p}=.000\right)$, relaciones sociales entre dirección y empleados $\left(\mathrm{F}_{(2,77)}=7,533, \mathrm{p}=.001\right)$, forma en que se dirige la sección $\left(\mathrm{F}_{(2,77)}=5,083, \mathrm{p}=.008\right)$, y en la estabilidad del empleo $\left(\mathrm{F}_{(2,77)}=8,005\right.$, $\mathrm{p}=.001)$,

- Igualmente existen diferencias significativas en el total de factores internos, factores externos y total (tabla 5).

Tabla 5. Comparación por tipo de contrato

\begin{tabular}{|l|c|c|c|}
\hline TIPO CONTRATO & F. interno & F. externos & Total \\
\hline Funcionario & $\mathbf{3 1 , 6}$ & $\mathbf{3 7 , 6}$ & $\mathbf{6 9 , 2}$ \\
\hline Contratado & $\mathbf{3 4 , 0}$ & $\mathbf{3 9 , 7}$ & $\mathbf{7 3 , 8}$ \\
\hline Laboral & $\mathbf{2 7 , 3}$ & $\mathbf{3 3 , 6}$ & $\mathbf{6 1}$ \\
& $\mathrm{F}(2,77)=5$, & $\mathrm{F}(2,77)=6$, & $\mathrm{F}(2,77)=6$, \\
& $763, \mathrm{p}=.005$ & $141, \mathrm{p}=.003$ & $740, \mathrm{p}=.002$ \\
\hline
\end{tabular}

- Los interinos obtienen menos satisfacción en el horario y en la estabilidad del empleo.

- Los laborales obtienen menos satisfacción en las condiciones físicas, libertad para escoger el método de trabajo, reconocimiento de una buena labor, cantidad de responsabilidad, salario, oportunidad para emplear sus capacidades, relaciones sociales entre dirección y empleados, oportunidad para pomocionar, forma en que se dirige la sección, atención que se le presta a sus sugerencias y las diferentes actividades que realiza en su trabajo.
Se observa que existen diferencias significativas entre salario, relaciones sociales entre dirección y trabajadores, forma de dirigir la sección y estabilidad laboral (factores externos) y reconocimiento a la buena labor, oportunidad para demostrar sus capacidades (factores internos), al igual que entre la puntuación total de factores internos/externos y la puntuación total de la satisfacción laboral.

Estos resultados son contradictorios con los datos encontrados en el estudio de Robina Ramirez (2002) donde el personal más satisfecho correspondía al laboral, mientras que los grupos de funcionarios alcanzan unas diferencias negativas supe- 
riores al 20\%, manifestando así las aspiraciones no satisfechas por la Administración, traducidos en la insatisfacción que experimentan sus empleados en la ejecución del trabajo.

Tampoco coincidimos con Loitegui (1990), quien encontró que los funcionarios son más críticos y están significativamente por debajo de los niveles medios de satisfacción en condiciones ambientales, tipo de trabajo que realizan, libertad e iniciativa en el trabajo y posibilidades de formación y solamente puntúan satisfactoriamente el salario. Así pues en este estudio los funcionarios demuestran estar más insatisfechos que los laborales fijos y éstos a su vez más insatisfechos que los contratados laborales temporales.

Pero si coincidimos con el resultado que presenta el grupo de contratados laborales con unos niveles de satisfacción significativamente superiores a los del grupo total en todos los factores analizados.

\section{Comparación de medias por escalas administrativas}

Dentro de las distintas escalas se señala los resultados siguientes:

- La escala que demuestra más satisfacción es la escala A-B/I-II, exceptuando en seguridad laboral, que es donde puntúan más bajo. Recordemos que muchos de estos puestos son de libre designación y por tanto pueden ser cesados, siendo su sentimiento de inseguridad en el puesto de trabajo.

- El grupo V es el que está más insatisfecho, pues exceptuando que se sien- ten muy a gusto con los compañeros de trabajo, en todos los demás ítems, puntúan con insatisfacción.

- El grupo C/III se siente insatisfecho en las condiciones físicas, libertad para escoger el método de trabajo, el trato con los compañeros, reconocimiento de una buena labor, la relación con el jefe más inmediato, relaciones sociales entre dirección y empleados, forma en que se dirige la sección, satisfacción en el horario y diferentes actividades que realiza en su trabajo.

- El grupo D/IV se muestra insatisfecho en los siguientes ítems: cantidad de responsabilidad, salario, oportunidad para emplear sus capacidades, oportunidad para pomocionar, , atención que se le presta a sus sugerencias.

- Cuando se buscan si existen diferencias estadísticamente significativas entre las diversas escalas laborales encontramos que se producen en los siguientes elementos: oportunidad para emplear sus capacidades $\left(\mathrm{X}^{2}=12,10, \mathrm{p}=.007\right)$, oportunidad para pomocionar $\left(\mathrm{X}^{2}=9,58, \mathrm{p}=.022\right)$, forma en que se dirige la sección $\left(\mathrm{X}^{2}=10,10, \mathrm{p}=.018\right)$, las diferentes actividades que realiza en su trabajo $\left(\mathrm{X}^{2}=9,48, \mathrm{p}=.024\right)$ y la estabilidad del empleo $\left(\mathrm{X}^{2}=10,99, \mathrm{p}=.012\right)$.

- También existen diferencias estadísticamente significativas en los factores interno, externo y el total. Se ha reseñar que la escala $V$ es la que más señala un nivel más alto de insatisfacción en todos los factores, tanto los de contenido como los higiénicos (tabla 6). 
Tabla 6. Comparación de factores internos/externos y total por tipos de escala

\begin{tabular}{|l|c|c|c|}
\hline ESCALAS & F. interno & F. externos & Total \\
\hline A-B/I-II & $\mathbf{5 6 . 6}$ & $\mathbf{5 1}$ & $\mathbf{5 4 . 9}$ \\
\hline C/III & 40.7 & $\mathbf{3 9 . 6}$ & $\mathbf{4 0 . 2}$ \\
\hline D/IV & $\mathbf{3 6 . 3}$ & $\mathbf{4 3}$ & $\mathbf{3 9 . 4}$ \\
\hline V & $\mathbf{2 2}$ & $\mathbf{2 2 . 3}$ & $\mathbf{2 0 . 8}$ \\
& $\mathbf{X}^{\mathbf{2}=11,92, p=.008}$ & $\mathrm{X}^{\mathbf{2}=7,89, p=.048}$ & $\mathrm{X}^{\mathbf{2}=10,79, \mathbf{p}=.013}$ \\
\hline
\end{tabular}

Nuestro datos no coinciden con los datos del trabajo de Robina Ramirez (2002), donde los empleados de los grupos II y IV (escala laboral) se hallan satisfechos especialmente en aquellos factores como la capacidad de asumir responsabilidades, $\mathrm{y}$ la posibilidad de participar en la toma de decisiones, mientras que en la escala A, B y $\mathrm{D}$ de funcionarios se encuentran levemente insatisfechos en casi todos los ítems. En los grupos de funcionario $\mathrm{C}$ y $\mathrm{E}$ esas diferencias negativa se presentan más acusadas en los elementos que tienen que ver con la orientación motivacional de la Administración, como por ejemplo, posibilidad de introducir ideas nuevas, contar con un trabajo interesante, asumir responsabilidades y participar en las decisiones.

En la escala II y I son los que está más satisfechos y destacando como elemento principal el sueldo, pero no valoran especialmente las relaciones con sus compañeros, ni la supervisión, ni la formación realizada por la Administración.

Se deberá investigar con mayor profundidad el descontento que se producen entre el personal laboral, para intentar minimizarlo.

\section{Comparación de medias por antigüedad en la Universidad}

En general, los trabajadores de más de
8 años de antigüedad se muestran más satisfechos que los que llevan menos años.

Los de mayor antigüedad están menos satisfechos con condiciones físicas, con los compañeros y en la relación con el jefe más inmediato.

Los de menor antigüedad están menos satisfechos con reconocimiento de una buena labor, cantidad de responsabilidad, salario, oportunidad para emplear sus capacidades, relaciones sociales entre dirección y empleados, oportunidad para pomocionar, forma en que se dirige la sección, atención que se le presta a sus sugerencias, satisfacción en el horario, las diferentes actividades que realiza en su trabajo y en la estabilidad del empleo.

A nivel de diferencias estadísticamente significativas, aparecen en los ítems siguientes: trato con los compañeros ( $\mathrm{t}(78)$ $=2,671, \mathrm{p}=.009)$, oportunidad para emplear sus capacidades $(\mathrm{t}(78)=-2,153, \mathrm{p}=.0 .34)$, oportunidad para pomocionar $(\mathrm{t}(78)=-$ $2,264, \mathrm{p}=0,26)$, satisfacción en el horario $(\mathrm{t}$ $(78)=-2,060, p=.043)$ y en la estabilidad del empleo $(\mathrm{t}(78)=-3,269, \mathrm{p}=.002)$.

Las medias de los factores internos, externos y total mantienen la misma tónica anterior, siendo el grupo con más antigüedad el que obtiene puntuaciones más altas, es decir es un grupo más satisfecho 
laboralmente, pero dichas diferencias no son significativas (tabla 7). desarrollo de todos los procesos donde intervienen el recurso humano. Las defi-

Tabla 7. Comparación de factores internos/externos y total por antigüedad

\begin{tabular}{|l|c|c|c|}
\hline ANTIGUEDAD & F. interno & F. externos & Total \\
\hline Menos de 8 años & 29.6 & 36.3 & 65.9 \\
\hline Más de 8 años & 31.6 & 37.2 & 68.9 \\
\hline
\end{tabular}

Según la teoría del ajuste laboral cabe esperar una relación positiva entre antigüedad y satisfacción laboral; al igual que se observa en el estudio de Lucas Marín (1981) donde demostró que los mayores niveles de satisfacción se alcanzan en relación a algunas características del puesto como: la relación con los compañeros, el trato con los subordinados y el tipo de salario. En nuestros resultados si encontramos que los mayores niveles de satisfacción, sobre todo en los factores internos se dan en los trabajadores de mayor antigüedad, pero los datos no son estadísticamente significativos.

Otros trabajos no han encontrado un vínculo estadísticamente significativo entre ambas variables (Clark, Oswald, y Warr, 1997; Freeman, 1980). Cuando el estudio se inscribe a trabajadores jóvenes (Belfield y Harris, 2002), el signo encontrado es negativo, lo que podría estar relacionado con la estrategia óptima de búsqueda de este colectivo. En el trabajo de Rodríguez y Prieto (2007) señalan que los trabajadores más jóvenes son los que tienen peores condiciones laborales y por tanto generan mayores niveles de insatisfacción.

\section{CONCLUSIONES}

Las condiciones de trabajo constituyen un elemento de gran importancia para el ciencias en este sentido puede ser causa de insatisfacción laboral. De ahí la importancia de medir la percepción de los empleados con respecto a sus condiciones de trabajo.

Los datos nos señalan que, en términos generales, la muestra del Personal de Administración y Servicios se halla satisfecha con sus compañeros de trabajo, el horario de trabajo, la estabilidad laboral que tiene, la libertad de procedimiento que se le ofrece para llevar a cabo su trabajo de la forma que piense que es más efectiva, la cantidad de responsabilidad que se le asigna y que en su puesto de trabajo pueda realizar diferentes actividades, puesto que más de dos tercios de la muestra así lo señalan. Esto nos habla de que prefieren una organización descentralizada con una buena estructura horizontal y cierto grado de autonomía.

Respecto a las distintas variables se observa que las mujeres están más satisfechas que los hombres, al igual que los trabajadores de mayor edad y lo que llevan más tiempo en la universidad; respecto al tipo de contrato, el interino es el que más satisfacción muestra, mientras que los laborales los que menos. Son las escalas V la más insatisfecha en contraste con las escalas A-I que son las más satisfechas.

Podemos afirma que los resultados muestran que las aspiraciones del PAS 
están satisfechas en la mayoría, pero atendiendo a la distribución de los factores motivantes propuestos por Herzberg, se observa que factores como las relaciones sociales con mando intermedios y la forma que tienen los superiores de dirigir la sección o el área son las principales preocupaciones del PAS y se encuentran insatisfechos con ellos. La importancia concedida por el PAS a cada uno de los aspectos que integran la relación se traduce en un deseo de ser dirigidos con competencia y preparación, pero además reclaman un establecimiento de un sistema de comunicación vertical que sea fluido, empático y bidireccional.

Sería interesante seguir investigando las diferencias de satisfacción que existen entre funcionario y laborales fijos, para poder determinar unas actuaciones que reduzcan dicha insatisfacción.

Las acciones de mejora que se podrían plantear pueden ser del tipo de desarrollo organizacional, así la dirección debe promover una estrategia para conseguir un cambio planificado y una mejora organizacional. En la literatura se puede encontrar númerosos ejemplos en que la promoción de determinados climas ha producido efectos positivos en la mejora de la organización. Básicamente la intervención sobre el clima incluye programas de entrenamiento y perfeccionamiento promovidos por la dirección y basadas en dinámicas grupales. En el trabajo de Ramis, Manassero, Ferrer, y García (2007) .queda patente la importancia de las habilidades comunicativas de los mandos intermedios y cómo influye en la satisfacción y a autoeficacia de sus colaboradores.

Finalmente, señalar que aunque el concepto de satisfacción laboral está definido de forma imprecisa y no es una medida de calidad de los trabajos, sino una comparación subjetiva de dichos trabajos por parte de los trabajadores, es importante seguir trabajando la satisfacción laboral pues parece claro la necesidad de mejorar la satisfacción como condición previa a cualquier otra medida de la empresa para mejorar su funcionamiento y su producción, pues es sabido que la insatisfacción lleva a fuertes actitudes negativas hacia la empresa, hacia sus decisiones y hacia todo lo que ella signifique.

\section{REFERENCIAS BIBLIOGRÁFICAS}

Arnold, J., Robertson, I y Cooper, C. (1991). Work psychology understanding human behavior in the work place. London: Pitman Publishing

Beer, M. (1964). Organizational size and job satisfaction. Academy of Management Journal, 7, 34-44

Belfield, C.R. y Harris, R.D.F. (2002). How well do theories of job matching explain variations in job satisfaction across education levels? Evidence for UK graduates. Applied Economics, 34, 535548.

Bravo, M. J. (1992). La satisfacción laboral en los profesionales sanitarios. Elaboración de un instrumento de evolución. Tesis de Licenciatura. Valencia.

Castillo Castillo, J. (1968). Satisfacción en el trabajo de los trabajadores andaluces. Anales de Sociología, 4, 4-5.

Clark, A.E. (1997). Job satisfaction and gender: Why are women so happy at work?. Labour Economics, 4, 341-372. 
Estudio comparativo de la satisfacción laboral en el personal de administración

Clark, A.E. y Oswald, A.J. (1994): Unhappiness and unemployment. Economic Journal, 104, 648-659.

Clark, A.E. y Oswald, A, .J. (1996). Satisfaction and comparison income. Journal of Public Economics 61, 65-71.

Clark, A.E., Oswald, A.J. y Warr, P.B. (1997). Is job satisfaction U-shaped in age?. Journal of Occupational and Organizational Psychology, 69, 57-81.

Cook, J. D. Hepwoorth, S., Wall, T. D. y Warr, P (1981).The experience of work. London: Academic Press.

Crites, J.O. (1969). Vocational Psychology. The study of vocational behavior and development. Nueva York: McGrawHill.

Cuadrado , I. Y Morales , J. F. (2007). Algunas claves sobre el techo de cristal en las organizaciones. Revista de Psicología del Trabajo y de las Organizaciones. 23 (2), 183-202.

Faragher, E. Cass, M y Cooper, C. (2005). The relationship between job satisfaction and health: a meta-analysis. Occupant Enviromente Medical, 20, 105-112.

Freeman, R. (1980). The exit-voice tradoff in the labor market:unionism, job tenure, quits and separations. Quarterly Journal of Economic, 94, 64-74.

Gamero Burón, C. (2003). Análisis Económico de la Satisfacción Laboral. Tesis Doctoral. Málaga.

Griffin, R. W y Bateman, T. S. (1986). Job satisfacation and organizacional commitment. En C. L. Cooper y I. Robertson (eds.). International review Industrial and Organizational Psychology. (147-189). New York: John Wiley and sons.

Groot, W. y Maassen van den Brink, H. (1998). Job satisfaction, wages and allocation of men and women, TSER/STT Working Papers, WP-10-98.

Harmon, J., Scotti, D. Behson, S. Farias, G., Petzel, R. Y Neuman, J. (2003). Effects of high-involment work systems on emplyee satisfaction and service cost in veterans healthcare. Journal Health Manag. 48, 393-406.

Harpaz, I. (1983). Job satisfaction. Theoretical perspectives and a longitudinal analysis. Nueva York: Libra Publishers.

Herzberg, F., Mausner, B. y Snyderman, B. B. (1959). The motivation to work. Nueva York:John Wiley \& Sons.

Kaiser, L.C. (2002). Job satisfaction: A comparison of standard, non-standard, and self- employment patterns across Europe with a special note to the gender/job satisfaction paradox. EPAG Working Papers, 27, Colchester: University of Essex.

Leonardi, R y Sloane, P. (2000). Measuring the quality of jobs:promotions porspects low pay and job satisfaction. Paper presented at Lower Conference on Low Pay, High Pay and the Determinants of Earnings Mobility in the European Union and the United States, University of Aberdeen, 17-18 November, 2000.

Locke, E. L. (1976). The natura of job satisfaction. In M. D. Dunnette (Ed.). Handbook of Organizational and Insdustrial Psychology. (80-114). Chicago: Rand. Mc.Nally. 
Loitegui Aldaz, J. (1990). Determinantes de la satisfacción laboral en empleados de la Administración Foral de Navarra. Tesis doctoral. Universidad Complutense.

Long, A. (2005). Happily ever after? A study of job satisfaction in Australia. The Economic Record, 81, 303-321

Lucas Marín, L (1981). .Una aproximación sociológica al estudio de la satisfacción en el trabajo en la provincia de Santander. REIS, 13, 99-100.

Newbury, D y Kamili, F. (2001). Psychology stress, anxiety, depression, job satisfaction and personality characteristisc in peregistrarion house officers. Postgrad Medical Journal, 77, 109-111.

Peiró, J. M. (1984).Psicología de la organización. Madrid: UNED.

Pérez , J. y Fidalgo M. (1994). NTP 394: Satisfacción laboral: escala general de satisfacción. Madrid: INSHT.

Quinn, R. P., Staines, G. L., y McCculloug, M. R.(1974). Job satisfaction: Is there a Trend?. Washington: U. S. Department of Labor.

Quintero, L., Biela, R., Barrera, A. y Campo A. (2007). Análisis factorial exploratorio de la escala de satisfacción laboral en empleados de un hospital psiquiátrico de Bucarmanga, Colombia. Revista Facultad de Medicina Universidad Nacional de Colombia. 55, 24-30.

Ramis, C. ,Manassero, A., Ferrer, V.A., y García , E. (2007). ¡No es fácil ser un buen jefe/a! Influencia de las habilidades comunicativas de la dirección sobre la motivación, la autoeficacia y la satisfacción de sus equipos de trabajo. Revista de
Psicología del Trabajo y de las Organizaciones. 23 (2), 161-181

Renaud, S. (2002). Rethinking the union membership/job satisfaction relationship. Some empirical evidence in Canada. Internacional Journal of Manpower, 23, 137-150

Robina Ramirez, R. (2002). Condicionantes sociolaborales de los empleados públicos: motivación y satisfacción laboral en la Administrción Regional de Extremadura. Tesis doctoral. Universidad de Extremadura.

Rodríguez, C. y Prieto J. (2007). Efecto de la afiliación sindical sobre la satisfacción laboral de los trabajadores en el caso español. XVI Jornadas de la Asociación de Economía de la Educación. Universidad de las Palmas de Gran Canarias. http//www.congresos.ulpgc.es/aeet_aede (consultado en febrero de 2008).

Salancik, G y Pfeffer, J. (1977). An examination of need-satisfaction models of job attitudes. Administrative Science Quarterly, 22, 427-456.

Schmitt, N y Loher (1984). Development of school satisfaction measures for NASSP task force on climate. Reston, VA: National Association for Secondary School Principals.

Sloane, P.J. y Williams, H. (2000). Job satisfaction, comparison earnings and gender. Labour, 14. 473-501.

Smith, P., Kendall, L y Hulin, C. (1969). The measurement of satisfaction in work and retirement. Chicago: Rand McNally.

Sousa-Poza, A. y Henneberger, F. (2002). Analyzing job mobility with job 
turnover intentions: An international comparative study. Presentado en 7th Annual Meeting of Society of Labor Economists, 3.4 May, Baltimore, USA.

Sousa-Poza, A. y Sousa-Poza, A.A. (2000). Taking another look at the gender/job- satisfaction paradox. Kyklos, 53, 135-152.

Tezanos, J., Lopez Aparicio, J., Rodríguez, J. y Domínguez, R. (1973). Las nиevas clases medias. Conflicto y conciencia de clase entre los empleados de la banca. Madrid: Edicusa.

Virtanen, M., Kivimaki, M., Elovainio, M, Vahtera, J. y Ferrie, J. (2003). From insecure to secure employment: changes in work, health, health relate behavoiurs and sickness absence. Occup. Enviro Med., 60,948-953.

Vroom, V (1964).Work and motivation. New Cork: Wiley.

Warr, Cook y Wall. (1979). Scales for the measurement of some work attitudes and aspects of psychological well-being. Journal of Occupational Psychology, 52, 129-148.

Warr, P. B. y Routledge, T. (1969). An opinion scale for the study of managers' job satisfaction. Occupational Psychology, 43, 95-109.

Weis, D. J.; Dawis, R. V.; England, G. W. y Lofquist, L. H. (1967). Manual for the Minnesota Satisfaction Questionnaire, Minneapolis: Industrial Relationship Center. University of Minnesota.

Wieclaw, J., Agerbo, E. Mortesen, P. y Bonde, J. (2005). Occupational risk of affective and stress-related disorders in the Danish workforce. Scan. Journal Environ Health, 31, 343-351.

Wooden, M. y Warren, D. (2004). Nonstandard employement and job satisfaction: Evidence from the HILDA survey. The Journal of Industrial Relations, 46, 275-297. 